\title{
A HYPERBOLIC UNIVERSAL OPERATOR COMMUTING WITH A COMPACT OPERATOR
}

\author{
CARL C. COWEN AND EVA A. GALLARDO-GUTIÉRREZ
}

\begin{abstract}
A Hilbert space operator is called universal (in the sense of Rota) if every operator on the Hilbert space is similar to a multiple of the restriction of the universal operator to one of its invariant subspaces. We exhibit an analytic Toeplitz operator whose adjoint is universal in the sense of Rota and commutes with a non-trivial, quasinilpotent, injective, compact operator with dense range, but unlike other examples, it acts on the Bergman space instead of the Hardy space and this operator is associated with a 'hyperbolic' composition operator.
\end{abstract}

\section{INTRODUCTION}

The notion of models for linear bounded operators acting on separable infinite dimensional Hilbert spaces $\mathcal{H}$ was introduced by Rota [21] in the sixties by proving the existence of linear bounded operators $U$ which models every separable infinite dimensional Hilbert space operator through its invariant subspaces.

More precisely, let $U$ be a bounded operator on $\mathcal{H}$, and denote by $\mathcal{B}(\mathcal{H})$ the algebra of bounded operators on $\mathcal{H}$. The operator $U$ is said to be universal for $\mathcal{H}$ if for each non-zero bounded operator $T$ on $\mathcal{H}$, there is an invariant subspace $M$ for $U$ and a non-zero number $\lambda$ such that $\lambda T$ is similar to $\left.U\right|_{M}$, that is, there is a linear isomorphism $S$ of $\mathcal{H}$ onto $M$ such that $S(\lambda T)=U S$.

Observe that if $U$ is a universal operator for a separable, infinite dimensional Hilbert space $\mathcal{H}$, then every bounded operator on $\mathcal{H}$ has an invariant subspace if and only if every non-zero subspace $M$ of $\mathcal{H}$ of dimension greater than 1 that is invariant for $U$ has a non-zero, proper subspace $M_{0}$ such that $M_{0}$ is also invariant for $U$. Therefore, understanding the invariant subspaces for any linear bounded operator $T$ on $\mathcal{H}$ becomes a question of understanding the invariant subspaces of the universal operator $U$.

Universal operators have attracted the attention of many operator theorists for the last few decades, mainly in connection with the Invariant Subspace Problem (see [2, Chapter 7] for references). The "standard" example of universal operator is the adjoint of a shift of infinity multiplicity, $S^{*}$, acting on a separable, infinite dimensional Hilbert space. Recently, in [8], the authors introduced a universal operator acting on $H^{2}=H^{2}(\mathbb{D})$ satisfying a rather surprising property: it commutes with a non-trivial, quasinilpotent, injective, compact operator with dense range, a property no universal operator like $S^{*}$ has.

Date: 29 November 2016.

2010 Mathematics Subject Classification. Primary: 47A15 Secondary: 47B35, 47B33, 47B38.

Key words and phrases. Invariant subspace, Bergman space, composition operator, Toeplitz operator, weighted composition operator, similarity, Rota's universal operators.

The first author was funded by Simons Foundation Collaboration Grant 358080 and the second author was partially supported by Plan Nacional I+D grant no. I+D MTM2013-42105-P and MTM2016-77710-P. The first author thanks the Departamento de Análisis Matemático, Facultad de Matemáticas, Universidad Complutense de Madrid for the hospitality shown him during summer 2015 and fall 2016 that made this work possible. 
The example provided in [8] (see also [5]) was accomplished in the classical Hardy space $H^{2}$, where the universal operator is the adjoint of an analytic Toeplitz operator $T_{\phi}^{*}$ and the compact operator is the adjoint of a compact weighted composition operator $W_{\psi, J}$ on $H^{2}$. Recall that if $\psi$ and $J$ are analytic maps of the unit disc $\mathbb{D}$, with $J(\mathbb{D}) \subset \mathbb{D}$, the weighted composition operator $W_{\psi, J}$ is defined by

$$
\left(W_{\psi, J} f\right)(z)=\psi(z) f(J(z))
$$

While $W_{\psi, J}$ is not always a bounded operator on $H^{2}$, if $\psi$ is a bounded analytic function on $\mathbb{D}$, then $W_{\psi, J}=T_{\psi} C_{J}$, where $T_{\psi}$ is a bounded analytic Toeplitz operator and $C_{J}$ is the composition operator induced by $J$, i.e. $\left(C_{J} f\right)(z)=f(J(z))$ for $f \in H^{2}$. Since all such composition operators $C_{J}$ and analytic Toeplitz operators $T_{\psi}$ are known to be bounded ([10], for example), the weighted composition operator $W_{\psi, J}$ is bounded on $H^{2}$.

The aim of this paper is taking the study of universal operators commuting with non-trivial, quasinilpotent, injective compact operators further by exhibiting a universal operator $T_{\phi}^{*}$ acting on the Bergman space, $A^{2}(\mathbb{D})=A^{2}$, commuting with the adjoint of a weighted composition operator $W_{\psi, J}=T_{\psi} C_{J}$ and for which the associated composition operator is 'hyperbolic'. As in the Hardy space $H^{2}$, if $J$ is an analytic map of the unit disk into itself, the composition operator $C_{J}$ on $A^{2}$ is the bounded operator for which $\left(C_{J} f\right)(z)=f(J(z))$. In our example, the relevant map $J$ of the disk to itself has a fixed point at 1 and $0<J^{\prime}(1)<1$ which means it is in the 'half-plane dilation case' of the model described in [6, pg. 70] or in [10, pg. 71] (also called 'hyperbolic' by some authors). In this example, for each $\alpha$ in $\mathbb{D}$, the iterates $J_{n}(\alpha)$ are an interpolating sequence and converge to 1 non-tangentially. While all separable, infinite dimensional Hilbert spaces are 'the same', the Toeplitz operators and composition operators $T_{\phi}, T_{\psi}$, and $C_{J}$ acting on the Hardy space $H^{2}$ and the Bergman space $A^{2}$, are quite different. We hope and expect that the easier to understand hyperbolic composition operator, $C_{J}$, and more complex Hilbert space, $A^{2}$, will allow a more careful analysis of invariant subspaces for these operators than previous examples do.

As noted above, the best known example of a universal operator is the adjoint of a unilateral shift of infinite multiplicity: for example, suppose $S$ is an analytic Toeplitz operator on the Hardy space $H^{2}$ whose symbol is a singular inner function or infinite Blaschke product. In this case, $S$ is an isometric operator and $S^{*}$ has infinite dimensional kernel and maps $H^{2}$ onto $H^{2}$, so $S^{*}$ is a universal operator by the Caradus Theorem (Theorem 1). Another widely known example of a universal operator was presented in the mid-1980's by Nordgren, Rosenthal and Wintrobe [19, 20] who proved that if $\varphi$ is a hyperbolic automorphism of the unit disc and $\mu$ is in the interior of the spectrum of the composition operator $C_{\varphi}$ acting on the classical Hardy space $H^{2}$, then $C_{\varphi}-\mu I$ is a universal operator on $H^{2}$. However, these operators cannot commute with any non-zero compact operators (see [3, Thm. 10], [4], and [7]). Because Lomonosov's Theorem [17] connects commuting with a non-trivial compact operator with the existence of invariant subspaces, it seems reasonable that a universal operator that commutes with a non-trivial compact operator might be helpful in proving the existence of invariant subspaces.

Concerning the structure of this paper, in Section 2, we first state the Caradus Theorem [1] that gives a sufficient condition for an operator to be universal in the sense of Rota [21]. Then, on the separable, infinite dimensional Hilbert space $A^{2}$, we construct an analytic Toeplitz operator and use the Caradus Theorem to prove that the adjoint of the analytic Toeplitz operator is universal. In Section 3, we construct a weighted composition operator and we prove the main theorem of the paper, that is, its adjoint is an injective, quasinilpotent, compact operator with dense range that 
commutes with the universal operator just constructed. The main tool in showing that the weighted composition operator is compact is Corollary 2 of Cučković and Zhao's paper [11] (see also [14, Proposition 3.1, (iii)]).

\section{A New Example of a Universal Operator}

The major work in this paper is set in the classical Bergman Hilbert space, $A^{2}$. Of course, because any two separable, infinite dimensional complex Hilbert spaces are isometrically isomorphic, our choice of $A^{2}$ is not limiting in any way. The space $A^{2}$ can be described in several ways:

$$
\begin{aligned}
A^{2} & =\left\{f \text { analytic in } \mathbb{D}: f(z)=\sum_{n=0}^{\infty} a_{n} z^{n} \text { where }\|f\|^{2}=\sum_{n=0}^{\infty} \frac{\left|a_{n}\right|^{2}}{n+1}<\infty\right\} \\
& =\left\{f \text { analytic in } \mathbb{D}:\|f\|^{2}=\int_{\mathbb{D}}|f(z)|^{2} \frac{d A(z)}{\pi}<\infty\right\}
\end{aligned}
$$

In each case, the norms for $f$ are the same. The space $A^{2}$ is a "Hilbert space of analytic functions" in the sense of [10]. In particular, this means that for $f$ in $A^{2}$, the map $f \mapsto f(\alpha)$ is a continuous linear functional for each $\alpha$ in the unit disk and $\left\langle f, K_{\alpha}\right\rangle=f(\alpha)$ where $K_{\alpha}(z)=(1-\bar{\alpha} z)^{-2}$, the kernel function for $\alpha$.

One of the main feature of $A^{2}$ is that, contrary to the Hardy space, there exist functions $f \in A^{2}$ having no radial limits a.e. on the boundary $\partial \mathbb{D}$. Moreover, the structure of the lattice of closed invariant subspaces for the shift operator has never been completely described and is known to be extremely complicated. We refer to the monographs [12] and [15] for more on the subject.

Nevertheless, the following theorem gives is a sufficient condition to provide universal operators on any Hilbert space.

Theorem 1. (Caradus [1, p. 527] or see [2, p. 214]) If $\mathcal{H}$ is a separable Hilbert space and $U$ is a bounded operator on $\mathcal{H}$ such that:

(1) The null space of $U$ is infinite dimensional.

(2) The range of $U$ is $\mathcal{H}$.

then $U$ is universal for $\mathcal{H}$.

We begin by defining the classes of operators we want to use, analytic Toeplitz operators and weighted composition operators. For $\phi$ a bounded analytic function on the unit disk, that is, $\phi$ is in $H^{\infty}(\mathbb{D})$, the analytic Toeplitz operator, $T_{\phi}$, on $A^{2}$ is the operator defined by $\left(T_{\phi} h\right)(z)=\phi(z) h(z)$ for $h$ in $A^{2}$. For $\phi$ a bounded analytic function on the disk, $T_{\phi}$ is a bounded operator on $A^{2}$ and it is easy to prove that $\left\|T_{\phi}\right\|=\|\phi\|_{\infty}$.

For $J$ an analytic map of the unit disk into itself, the composition operator, $C_{J}$, on $A^{2}$ is the operator defined by $\left(C_{J} h\right)(z)=h(J(z))$. The boundedness of $C_{J}$ for any analytic function $J$ mapping the unit disk into itself is a consequence of the Littlewood Subordination Theorem [16] (or see [10, pp. $30 \& 117])$. If $\psi$ is in $H^{\infty}(\mathbb{D})$ and $J$ is an analytic map of the disk to itself, the weighted composition operator, $W_{\psi, J}$, on $A^{2}$ is the operator $W_{\psi, J}=T_{\psi} C_{J}$, also a bounded operator.

To begin our construction, for $z$ in $\mathbb{D}$, let $\sigma(z)=-i \log (1-z)$ where we take the branch of the logarithm that is analytic on $\mathbb{C} \backslash(-\infty, 0]$ and satisfies $\log (1)=0$. The map $\sigma$ is a univalent map on the unit disk and Figure 1 illustrates the set $\sigma(\mathbb{D})$. 
Now, we define the bounded analytic function $\phi$ by

$$
\phi(z)=e^{\sigma(z)}-e^{\sigma(0)}=e^{\sigma(z)}-1
$$

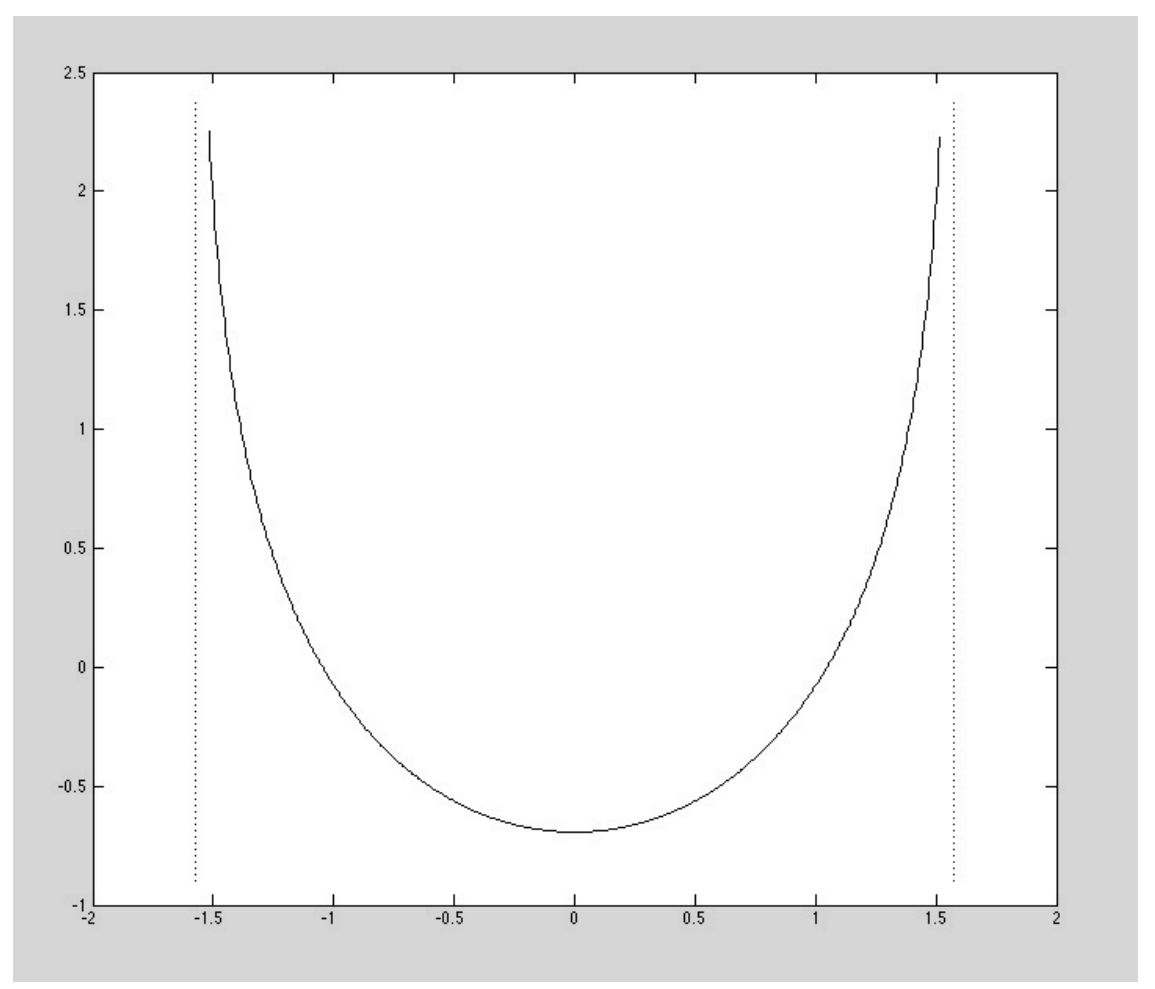

Figure 1. The set $\sigma(\partial \mathbb{D})$ with $\operatorname{Re}(w)= \pm \pi / 2$.

It will be helpful to point out some of the properties of $\sigma$ and $\phi$. We will use the set $\Gamma=\left\{e^{i \theta}: 0<\theta<2 \pi\right\}$, the unit circle except 1 , in this description.

[1] The map $z \mapsto 1-z$ takes 1 to 0 and the rest of the closed disk into the open right half plane, so we see that $\sigma$ is continuous on the closed disk except at $z=1$, that for $z$ in $\Gamma$, we have $-\pi / 2<\operatorname{Re}(\sigma(z))<\pi / 2$, and that $\sigma([-1,1))$ is the ray $\{i y: y \geq-\log (2)\}$, (see Figure 1). Also, $\sigma(-1)=-i \log (2), \lim _{r \rightarrow 1^{-}} \sigma(r)=+i \infty, \lim _{\theta \rightarrow 0^{+}} \operatorname{Re}\left(\sigma\left(e^{i \theta}\right)\right)=-\pi / 2$, and $\lim _{\theta \rightarrow 2 \pi^{-}} \operatorname{Re}\left(\sigma\left(e^{i \theta}\right)\right)=\pi / 2$. Moreover, $\sigma(0)=0$ is not on the curve $\sigma(\Gamma)$.

[2] For each positive number $t$ and for each $w$ in $\sigma(\mathbb{D})$, we see that $w+i t$ is also in $\sigma(\mathbb{D})$.

[3] The exponential function maps the strip $-\pi / 2<\operatorname{Re}(w)<\pi / 2$ onto the annulus $\left\{\zeta: e^{-\pi / 2}<|\zeta|<e^{\pi / 2}\right\}$ which has center at the origin and inner and outer radii $e^{-\pi / 2}$ and $e^{\pi / 2}$, indeed, this is precisely the (infinite-to-one) covering map of the strip onto the annulus. The function $e^{\sigma}$ maps the curve $\Gamma$ onto a curve spiraling out from unit circle at the center of the annulus and tending asymptotically to its inner and outer boundaries:

$$
\lim _{\theta \rightarrow 0^{+}}\left|e^{\sigma\left(e^{i \theta}\right)}\right|=e^{-\pi / 2} \text { and } \lim _{\theta \rightarrow 2 \pi^{-}}\left|e^{\sigma\left(e^{i \theta}\right)}\right|=e^{\pi / 2}
$$

In particular, because each vertical line $\left\{x_{0}+i y:-\infty<y<\infty\right\}$, for $-\pi / 2<x_{0}<\pi / 2$, intersects the curve $\sigma(\Gamma)$ exactly one point, for each $r$ with $e^{-\pi / 2}<r<e^{\pi / 2}$, the circle of radius $r$ centered at the origin intersects the curve $e^{\sigma(\Gamma)}$ in exactly one point. Moreover, the 


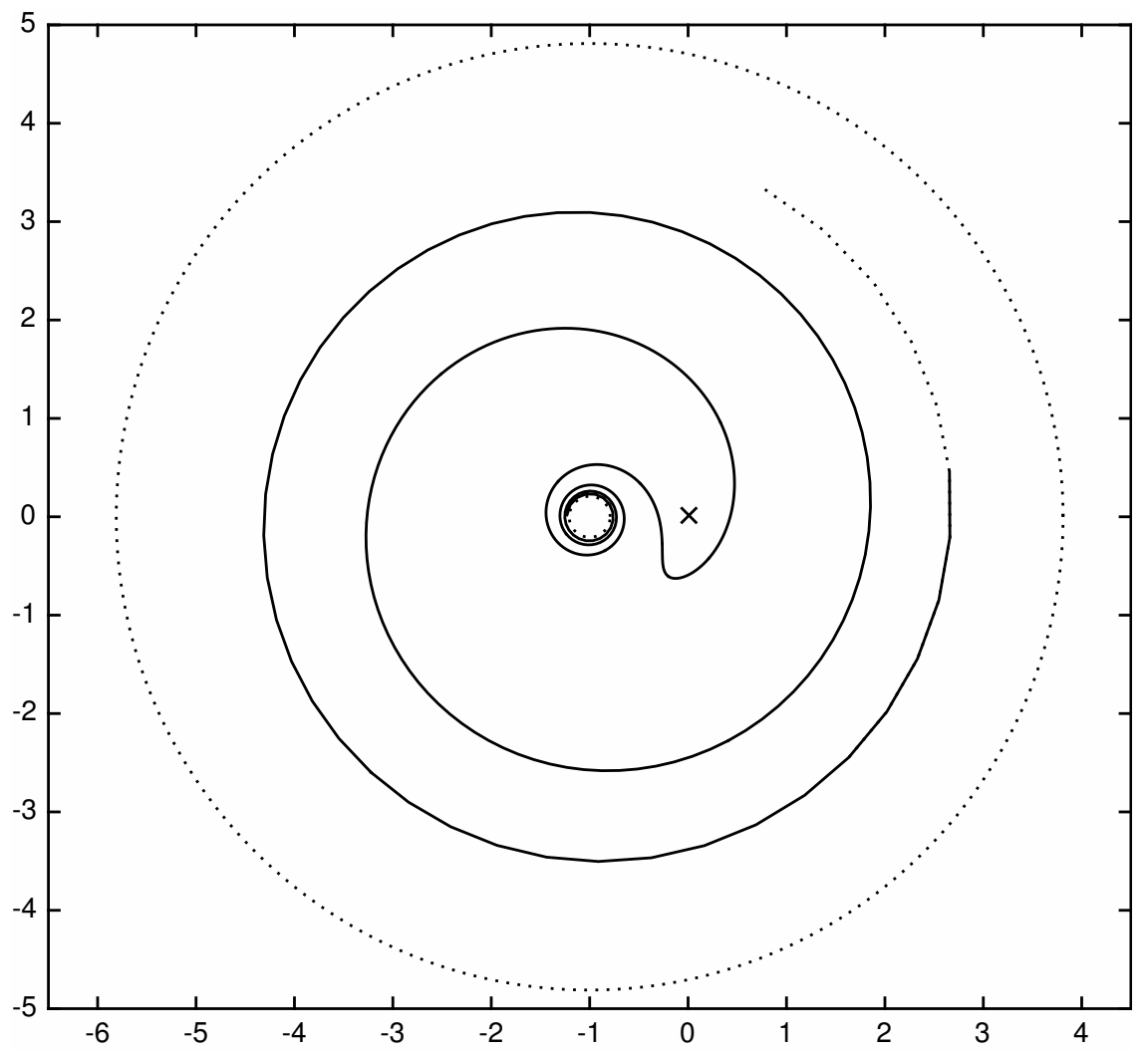

Figure 2. The set $\phi(\Gamma)$ in the annulus $\phi(\mathbb{D})$ including ' $\mathrm{x}$ ' at 0 .

closure of $e^{\sigma(\Gamma)}$ is the set $\sigma(\Gamma) \cup\left(e^{-\pi / 2} \partial \mathbb{D}\right) \cup\left(e^{\pi / 2} \partial \mathbb{D}\right)$ and the distance of $e^{\sigma(0)}=1$ from this closed set is positive.

[4] The function $e^{\sigma}$ is an infinite-to-one map of the unit disk, $\mathbb{D}$, onto the annulus $\left\{\zeta: e^{-\pi / 2}<|\zeta|<e^{\pi / 2}\right\}$.

Proof of [4]: For every $r$ with $e^{-\pi / 2}<r<e^{\pi / 2}$, by part [3] above, the curve $e^{\sigma(\Gamma)}$ intersects the circle of radius $r$ with center at 0 exactly once. This means there is $\theta_{r}$ with $0<\theta_{r}<2 \pi$ so that $\left|e^{\sigma\left(\theta_{r}\right)}\right|=r$. Now the ray $\left\{w=\sigma\left(\theta_{r}\right)+i y: y>0\right\}$ is contained in $\Omega=\sigma(\mathbb{D})$. Since the real parts of each of the numbers on this ray are the same, each point of the ray is mapped by the exponential function onto the circle of radius $r$. Since $z$ in the ray implies $z+2 n \pi i$ is on the ray for every positive integer $n$, the exponential function maps the ray infinite-to-one onto the circle. Since the union of these circles is $\left\{\zeta: e^{-\pi / 2}<|\zeta|<e^{\pi / 2}\right\}$, this proves [4].

[5] The function $\phi=e^{\sigma}-1$, defined in Equation (1), is bounded below on $\Gamma$, and therefore is an invertible function in $L^{\infty}(\partial \mathbb{D})$.

Proof of [5]: Clearly $\|\phi\|_{\infty} \leq 1+e^{\pi / 2}$ because $e^{\sigma(\Gamma)} \subset e^{\pi / 2} \mathbb{D}$. By [3] above, the distance from $e^{\sigma(\Gamma)}$ to 1 is positive, so the distance from $\phi(\Gamma)$ to 0 is positive, (see Figure 2). This means the function $1 / \phi$ is also in $L^{\infty}(\partial \mathbb{D})$ and $\phi$ is bounded below on $\Gamma$.

Lemma 2. Let $\phi$ be the function in $H^{\infty}(\mathbb{D})$ defined in Equation (1). There is a bounded operator $S$ on $A^{2}$ that is a left inverse for the analytic Toeplitz operator $T_{\phi}$. 
Proof. For $\sigma(z)=-i \log (1-z)$, the map $\phi(z)=e^{\sigma(z)}-1$ is a bounded analytic function on $\mathbb{D}$ and, by [5] above, $\phi$ is bounded below on $\Gamma=\left\{e^{i \theta}: 0<\theta<2 \pi\right\}$. An easy calculation gives

$$
\phi^{\prime}(z)=\sigma^{\prime}(z) e^{\sigma(z)}=\frac{i}{1-z} e^{\sigma(z)}
$$

which means that $\phi^{\prime}(z)$ is never 0 . Since $\phi^{\prime}(z) \neq 0$ on $\mathbb{D}$, Theorem 6 of [9] shows that the inner factor of $\phi$ is an interpolating Blaschke product, $B$. Since $\phi$ is bounded below on $\Gamma$, this means $\phi=B g$ where $g$ is an invertible function in $H^{\infty}(\mathbb{D})$.

Since the inner factor of $\phi$ is an interpolating Blaschke product, McDonald and Sundberg's paper [18, p. 609] shows that $T_{\phi}$ has closed range, that is, the subspace $\phi A^{2}$ is closed. Since analytic Toeplitz operators are injective, the closed graph theorem says there is a bounded operator $S$ on $A^{2}$ so that $S T_{\phi}=I$.

Corollary 3. The Toeplitz operator $T_{\phi}^{*}$ has a right inverse and $T_{\phi}^{*}$ maps $A^{2}$ onto itself.

Proof. From Lemma 2, we have $S T_{\phi}=I$, so $T_{\phi}^{*} S^{*}=\left(S T_{\phi}\right)^{*}=I$. This equality implies $T_{\phi}^{*}$ maps $A^{2}$ onto itself.

The following result is the first goal of this paper.

Theorem 4. If $\phi$ is the function defined in Equation (1), the Toeplitz operator $T_{\phi}^{*}$ is universal for $A^{2}$.

Proof. We use the Theorem of Caradus (Theorem 1 above, [1], or [2, p. 214]) to establish the result.

First, Corollary 3 shows that the range of $T_{\phi}^{*}$ is all of $A^{2}$.

For $n$ a non-negative integer, let $w_{n}=2 n \pi i$. Notice that each of these points is in $\Omega$. Since $\sigma$ is a Riemann map of $\mathbb{D}$ onto $\Omega$, we let $z_{n}=\sigma^{-1}\left(w_{n}\right)$. Although we do not need it here, $\left\{z_{n}\right\}$ is an interpolating sequence of real numbers in the disk that converges (non-tangentally) to 1 .

Now, writing $K_{z_{n}}$ for the kernel for evaluation of $A^{2}$ functions at $z_{n}$, we see

$$
T_{\phi}^{*}\left(K_{z_{n}}\right)=\overline{\phi\left(z_{n}\right)} K_{z_{n}}=\overline{\left(e^{\sigma\left(z_{n}\right)}-1\right)} K_{z_{n}}=\overline{\left(e^{2 n \pi i}-1\right)} K_{z_{n}}=0
$$

which means for each $n$, the functions $K_{z_{n}}$ are in the kernel of $T_{\phi}^{*}$. But the functions $K_{z_{n}}$ are linearly independent, so the kernel of $T_{\phi}^{*}$ is infinite dimensional.

By Caradus' Theorem, $T_{\phi}^{*}$ is a universal operator for $A^{2}$.

\section{A Compact Operator Commuting with the Universal Operator}

The goal of this section is to construct a compact weighted composition operator that commutes with the analytic Toeplitz operator $T_{\phi}$. The following lemma answers the question 'When does a weighted composition operator commute with an analytic Toeplitz operator?' This result is also Lemma 6 of [8].

Lemma 5. For $\phi$ and $\psi$ in $H^{\infty}$ and $J$ an analytic map of the unit disk into itself, the analytic Toeplitz operator $T_{\phi}$ commutes with the composition operator $C_{J}$ or the weighted composition operator $W_{\psi, J}$ if and only if $\phi \circ J=\phi$.

Proof. The cases $J$ constant or $\phi$ constant are trivial, so we assume neither is constant. It is easy to see that two analytic Toeplitz operators commute with each other. Since $W_{\psi, J}=T_{\psi} C_{J}$, it is enough to check the statement for $T_{\phi}$ and $C_{J}$. We have $\left(T_{\phi} C_{J} h\right)(z)=\phi(z) h(J(z))$ and $\left(C_{J} T_{\phi} h\right)(z)=\left(C_{J} \phi h\right)(z)=\phi(J(z)) h(J(z))$. These are equal, for all $z$ in $\mathbb{D}$, for a non-zero $h$ if and only if $\phi(z)=\phi(J(z))$. 
To create the desired compact weighted composition operator, we keep the lemma above in mind and define $J$ by

$$
J(z)=\sigma^{-1}(\sigma(z)+2 \pi i)
$$

where $\sigma$ is the map $\sigma(z)=-i \log (1-z)$ of the disk into the strip defined in Section 2. We note that $J$ is well defined because $\sigma$ is univalent on $\mathbb{D}$, and $J$ maps the disk into itself because $\sigma(\mathbb{D})$ is carried into itself by the translation $w \mapsto w+2 \pi i$.

From this definition, an easy calculation shows that $\phi \circ J=\phi$ :

$$
\phi(J(z))=e^{\sigma\left(\sigma^{-1}(\sigma(z)+2 \pi i)\right)}-e^{\sigma(0)}=e^{(\sigma(z)+2 \pi i)}-e^{\sigma(0)}=e^{\sigma(z)}-e^{\sigma(0)}=\phi(z)
$$

It will be helpful if we find a simpler expression for $J$, however. To begin, if $w=\sigma(z)=-i \log (1-z)$ then

$$
\begin{aligned}
i w & =\log (1-z) \\
e^{i w} & =1-z \\
\sigma^{-1}(w) & =z=1-e^{i w}
\end{aligned}
$$

Now

$$
\begin{aligned}
J(z) & =\sigma^{-1}(\sigma(z)+2 \pi i) \\
& =1-e^{i(-i \log (1-z)+2 \pi i)} \\
& =1-e^{(\log (1-z)-2 \pi)} \\
& =1-e^{-2 \pi}(1-z)=\left(1-e^{-2 \pi}\right)+e^{-2 \pi} z
\end{aligned}
$$

That is, $J$ is an affine map of the disk into itself that fixes 1 and maps the real axis to itself!

Lemma 6. For $J$ as in Equation (4), the operator $C_{J}$ has dense range in $A^{2}$.

Proof. Clearly, if $p$ is a polynomial of degree $d$, the form of $J$ guarantees that $C_{J}(p)$ is also a polynomial of degree $d$. This means that the images of $C_{J}\left(z^{k}\right)$ for $k=0, \cdots, n$ are linearly independent for any positive integer $n$. It follows that every polynomial is in the range of $C_{J}$, which means the range of $C_{J}$ is dense in $A^{2}$.

Lemma 7. For $J$ as in Equation (4), the function

$$
\frac{|1-J(z)|^{2}}{1-|J(z)|^{2}}
$$

with the value at $z=1$ assigned to be $e^{-2 \pi}\left(1-e^{-2 \pi}\right)^{-1}$, is continuous and non-zero on the closed unit disk.

Proof. From Equation (4), it is clear that $J$ maps the closed unit disk into itself and that the only point of the range of $J$ that lies on the unit circle is its fixed point at 1 . In particular, this means the only point of the closed disk at which $|1-J(z)|^{2}$ and $1-|J(z)|^{2}$ are zero is at $z=1$ and that the expression in (5) is indeterminate at $z=1$. Assigning the value of the expression to be $e^{-2 \pi}\left(1-e^{-2 \pi}\right)^{-1}$ at $z=1$ means that we have a well defined function on the closed unit disk that is never zero. Clearly, this expression is continuous at all points of the closed unit disk besides $z=1$, so we only need to show that the function is continuous at $z=1$ to prove the lemma.

For $J(z)=1-e^{-2 \pi}+e^{-2 \pi} z$, clearly $1-J(z)=e^{-2 \pi}(1-z)$. Writing $z=r e^{i \theta}$ for $0 \leq r \leq 1$ and $-\pi<\theta \leq \pi$, we see that

$$
|1-J(z)|^{2}=e^{-4 \pi}\left(1-r e^{i \theta}\right)\left(1-r e^{-i \theta}\right)=e^{-4 \pi}\left(1-2 r \cos (\theta)+r^{2}\right)
$$


is continuous and that $\lim _{r \rightarrow 1}|1-J(z)|^{2}=2 e^{-4 \pi}(1-\cos (\theta))$. Similarly, $|J(z)|^{2}=\left(1-e^{-2 \pi}+e^{-2 \pi} r e^{i \theta}\right)\left(1-e^{-2 \pi}+e^{-2 \pi} r e^{-i \theta}\right)=1-2 e^{-2 \pi}+e^{-4 \pi}+2\left(1-e^{-2 \pi}\right) e^{-2 \pi} r \cos (\theta)+e^{-4 \pi} r^{2}$

It follows that

$$
1-|J(z)|^{2}=e^{-2 \pi}\left(2-e^{-2 \pi}-2\left(1-e^{-2 \pi}\right) r \cos (\theta)-e^{-2 \pi} r^{2}\right)
$$

which is continuous and

$$
\lim _{r \rightarrow 1} 1-|J(z)|^{2}=e^{-2 \pi}\left(2-e^{-2 \pi}-2\left(1-e^{-2 \pi}\right) \cos (\theta)-e^{-2 \pi}\right)=2 e^{-2 \pi}\left(1-e^{-2 \pi}\right)(1-\cos (\theta))
$$

This means that for $\theta \neq 0$, we have

$$
\lim _{r \rightarrow 1} \frac{|1-J(z)|^{2}}{1-|J(z)|^{2}}=\frac{2 e^{-4 \pi}(1-\cos (\theta))}{2 e^{-2 \pi}\left(1-e^{-2 \pi}\right)(1-\cos (\theta))}=\frac{e^{-2 \pi}}{1-e^{-2 \pi}}
$$

Therefore, with the assignment of $e^{-2 \pi}\left(1-e^{-2 \pi}\right)$ for $z=1$, we have the continuity of the function in (5) on the closed unit disk.

With these definitions and the Lemmas of this section, we are ready to prove the following result, the main goal of this paper.

Theorem 8. If $J(z)=\left(1-e^{-2 \pi}\right)+e^{-2 \pi} z$ and $\psi(z)=(1-J(z))^{2}$, the operator $W_{\psi, J}^{*}$ is an injective, quasinilpotent, compact operator with dense range that commutes with the universal operator $T_{\phi}^{*}$.

Proof. Theorem 4 asserts that $T_{\phi}^{*}$ is a universal operator in the sense of Rota.

The operator $W_{\psi, J}=T_{\psi} C_{J}$ and since analytic Toeplitz operators and composition operators have nullspace (0), the operator $W_{\psi, J}$ is injective. By Lemma 6 , the operator $C_{J}$ has dense range. Since $1-J(z)=-e^{-2 \pi}(1-z)$ and $T_{1-z}$ is well known to have dense range, we see that $T_{\psi}$ has dense range and therefore $W_{\psi, J}$ has dense range as well. It follows that $W_{\psi, J}^{*}$ is also injective and has dense range.

By Lemma 5 and Equation (3), we see that $W_{\psi, J}$ and $T_{\phi}$ commute, so $W_{\psi, J}^{*}$ and $T_{\phi}^{*}$ commute.

By Corollary 2 of Čučković and Zhao's paper [11] (see also [14, Proposition 3.1, (iii)]) $W_{\psi, J}$ is compact on $A^{2}$ if and only if

$$
\lim _{|a| \rightarrow 1^{-}} \int_{\mathbb{D}} \frac{\left(1-|a|^{2}\right)^{2}|\psi(z)|^{2}}{|1-\bar{a} J(z)|^{4}} d A(z)=0
$$

To check this condition, we note that

$$
\begin{aligned}
\int_{\mathbb{D}} \frac{\left(1-|a|^{2}\right)^{2}|\psi(z)|^{2}}{|1-\bar{a} J(z)|^{4}} d A(z) & =\int_{\mathbb{D}} \frac{\left(1-|a|^{2}\right)^{2}|1-J(z)|^{4}}{|1-\bar{a} J(z)|^{4}} d A(z) \\
& =\int_{\mathbb{D}}\left(\frac{|1-J(z)|^{2}}{1-|J(z)|^{2}}\right)^{2}\left(\frac{\left(1-|a|^{2}\right)\left(1-|J(z)|^{2}\right)}{|1-\overline{J(z)} a|^{2}}\right)^{2} d A(z) \\
& =\int_{\mathbb{D}}\left(\frac{|1-J(z)|^{2}}{1-|J(z)|^{2}}\right)^{2}\left(1-\left|\frac{a-J(z)}{1-\overline{J(z)} a}\right|^{2}\right)^{2} d A(z)
\end{aligned}
$$

where the last equality follows from the identity:

$$
1-\left|\frac{\alpha-\beta}{1-\bar{\beta} \alpha}\right|^{2}=\frac{\left(1-\mid \alpha^{2}\right)\left(1-|\beta|^{2}\right)}{|1-\bar{\beta} \alpha|^{2}}, \quad(\alpha, \beta \in \mathbb{D})
$$


(see [13, Chapter 1], for instance).

Now, Lemma 7 shows that the first factor in the integral in Equation (6) is a continuous function on the closed disk that is never zero, which allows us to conclude that $|1-J(z)|^{4} /\left(1-|J(z)|^{2}\right)^{2}$ belongs to $L^{1}(\mathbb{D}, d A(z))$.

Notice that the second factor in the integral in Equation (6) is no greater than 1 for $a$ and $z$ in the unit disk: taking $z$ to be fixed, since $J(z)$ is a point of the disk, the resulting function of $a$ is the absolute value of an automorphism of the disk, so we see

$$
\left|\frac{a-J(z)}{1-\overline{J(z)} a}\right| \leq 1
$$

and we also see that, for each $z$ in $\mathbb{D}$,

$$
\lim _{|a| \rightarrow 1}\left|\frac{a-J(z)}{1-\overline{J(z)} a}\right|=1
$$

Therefore, by the Dominated Convergence Theorem of Lebesgue, the limit in Equation (6) is zero. This proves that $W_{\psi, J}$, and $W_{\psi, J}^{*}$, are compact on $A^{2}$.

Finally, because the compact operator $W_{\psi, J}$ commutes with the analytic Toeplitz operator $T_{\phi}$, if $\lambda \neq 0$ were an eigenvalue of $W_{\psi, J}$, then its eigenspace would be a finite dimensional invariant subspace for $T_{\phi}$ which cannot be true. Thus, $W_{\psi, J}$, and therefore $W_{\psi, J}^{*}$, are both quasinilpotent compact operators. This concludes the proof of Theorem 8.

Corollary 9. If $\eta$ is a bounded analytic function on the open unit disk for which the Toeplitz operator $T_{\eta}$ is invertible on $A^{2}$, then the operator $W_{\eta \psi, J}^{*}$ is an injective, quasinilpotent, compact operator with dense range that commutes with the universal operator $T_{\phi}^{*}$.

Proof. The operator $W_{\eta \psi, J}^{*}=W_{\psi, J}^{*} T_{\eta}^{*}$. Since $T_{\eta}^{*}$ is invertible and commutes with $T_{\phi}^{*}$ and $W_{\psi, J}^{*}$ is an injective compact operator that commutes with $T_{\phi}^{*}, W_{\eta \psi, J}^{*}$ has these properties as well. The quasinilpotence follows as in the proof of Theorem 8 .

This example of a universal operator that commutes with compact operators seems easier to use than previously known examples, and at the same time, operates on a more complex Hilbert space than $H^{2}$. It is hoped that these examples will be fruitful in studying invariant subspaces for general operators.

\section{REFERENCES}

[1] Caradus, S. R., Universal operators and invariant subspaces, Proc. Amer. Math. Soc. 23(1969), $526-527$.

[2] Chalendar, I. and Partington, J. R., Modern Approaches to the Invariant Subspace Problem, Cambridge University Press, 2011.

[3] Cowen, C. C., The commutant of an analytic Toeplitz operator, Trans. Amer. Math. Soc. 239(1978), 1-31.

[4] Cowen, C. C., The commutant of an analytic Toeplitz operator, II, Indiana Univ. Math. J. 29(1980), 1-12.

[5] Cowen, C. C., An analytic Toeplitz operator that commutes with a compact operator and a related class of Toeplitz operators, J. Funct. Anal. 36(1980), 169-184.

[6] Cowen, C. C., Iteration and the solution of functional equations for functions analytic in the unit disk, Trans. Amer. Math. Soc. 265(1981), 69-95.

[7] Cowen, C. C. and Gallardo-Gutiérrez, E. A., Unitary equivalence of one-parameter groups of Toeplitz and composition operators, J. Funct. Anal. 261(2011), 2641-2655.

[8] Cowen, C. C. and Gallardo-Gutiérrez, E. A., Rota's universal operators and invariant subspaces in Hilbert spaces, J. Funct. Anal. 271(2016), 1130-1149.

[9] Cowen, C. C., Gallardo-Gutiérrez, E. A., and Gorkin, P. , When is the inner factor of $f-f(a)$ an interpolating Blaschke product for all $a$ ?, preprint, 2016. 
[10] Cowen, C. C. and MacCluer, B. D., Composition Operators on Spaces of Analytic Functions, CRC Press, 1995.

[11] Čučković, Ž. and Zhao, R., Weighted composition operators on the Bergman space, J. Lond. Math. Soc. (2) 70(2004), 499-511.

[12] Duren, P. and Schuster, A. Bergman spaces, Mathematical Surveys and Monographs, 100, AMS 2004.

[13] Garnett, J. B., Bounded analytic functions, Revised first edition. Springer-Verlag, New York, 2007.

[14] Gallardo-Gutiérrez, E. A., Kumar, R. and Partington, J. P., Boundedness, compactness and Schatten-class membership of weighted composition operators, Integral Equations Operator Theory 67(2010), 467-479.

[15] Hedenmalm, H., Koremblu, B. and Zhu, K. Theory of Bergman Spaces, Graduate Texts in Mathematics 199, Springer-Verlag, 2000.

[16] Littlewood, J. E., On inequalities in the theory of functions, Proc. Lond. Math. Soc. 23(1925), 481-519.

[17] Lomonosov, V., On invariant subspaces of families of operators commuting with a completely continuous operator, Funktsional. Anal. i Prilozhen. 7(1973), 55-56. (Russian).

[18] McDonald, G. and Sundberg, C., Toeplitz operators on the disc, Indiana Univ. Math. J. 28(1979), 595-611.

[19] Nordgren, E. A., Rosenthal, P. and Wintrobe, F. S., Composition operators and the invariant subspace problem, C. R. Math. Acad. Sci. Soc. R. Can. 6(1984), 279-282.

[20] Nordgren, E. A., Rosenthal, P. and Wintrobe, F. S., Invertible composition operators on $H^{p}$, J. Funct. Anal. 73(1987), 324-344.

[21] Rota, G.-C., On models for linear operators, Comm. Pure Appl. Math. 13(1960), 469-472.

Department of Mathematical Sciences, Indiana University-Purdue University Indianapolis, INDIANAPOLIS, IN 46202, USA

E-mail address: ccowen@iupui.edu

Departamento de Análisis Matemático, Facultad de Matemáticas, Universidad Complutense de Madrid e ICMAT, Plaza de Ciencias № 3, 28040 Madrid, SPAIN.

E-mail address: eva.gallardo@mat.ucm.es 